\title{
New results on cut-off effects in spectroscopy with the fixed point action
}

\author{
Peter Hasenfratz ${ }^{a}$, K. Jimmy Juge ${ }^{b}$ and Ferenc Niedermayer ${ }^{a, c}$ \\ BGR (Bern-Graz-Regensburg) Collaboration \\ ${ }^{a}$ Institute for Theoretical Physics, University of Bern \\ Sidlerstrasse 5, CH-3012 Bern, Switzerland \\ ${ }^{b}$ School of Mathematics, Trinity College \\ Dublin 2, Ireland \\ ${ }^{c}$ On leave of absence from Eötvös University, HAS Research Group, \\ Budapest, Hungary
}

\begin{abstract}
Our study on the cut-off effects in quenched light hadron spectroscopy and pion scattering length with the fixed point action is extended by results obtained at a lattice spacing $a=0.102 \mathrm{fm}$ in a box of size $\mathrm{L}=1.8$ $\mathrm{fm}$. The cut-off effects are small, but clearly seen as the resolution is increased from $\mathrm{a}=0.153 \mathrm{fm}$ to $\mathrm{a}=0.102 \mathrm{fm}$. In the quark mass region where the errors are small and under control, our results on the APE plot lie close to the extrapolated numbers of the CP-PACS Collaboration.
\end{abstract}




\section{Introduction and summary}

In a recent paper [1], the BGR-Collaboration presented results on light hadron (down to $m_{\pi} / m_{\mathrm{N}} \approx 0.25$ ) quenched spectroscopy with two different actions: the parametrized fixed point (FP) action [2, 3] and the CI action which is a combination of the Lüscher-Weisz gauge action 4] with a chirally improved Dirac operator [5]. In this paper we extend the scaling analysis in ref. [1 on the FP action by adding new results on an $18^{3} \times 36$ lattice with lattice unit $a\left(r_{0}\right)=0.102 \mathrm{fm}$. This extension is necessary, since our scaling test in [1] referred to a small $L=1.2 \mathrm{fm}$ spatial size lattice with low statistics, while our large $L=2.4 \mathrm{fm}$ lattice results with good statistics could be compared with other simulations only. The new data have been used already in a scaling test on the $I=2, \pi \pi$ scattering length $[\underline{6}$. No cut-off effects were seen there, but it is difficult to measure the scattering length precisely. We need reliable information on the cut-off effects in the quenched approximation since these results help to choose the parameters of future full QCD simulations the BGR Collaboration is moving towards. In addition, this information might be helpful when comparing the results from different actions for consistency. Indeed, there exist unclarified inconsistencies between different works in light hadron spectroscopy. On the APE plot, for example, the CP-PACS 7] continuum extrapolated numbers lie significantly below the data obtained with most of the different improved actions. For a recent comparison of numerical data obtained with different actions see ref. 8 .

The parametrized FP Dirac operator lives on the hypercube, has 81 offsets, a large number of paths, uses all the elements of the Clifford algebra 3 and satisfies the Ginsparg-Wilson relation up to parametrization errors. The setup of the present simulation and the analysis follows closely those applied in ref.[1]. We generated 180 gauge configurations separated by 500 alternating Metropolis and pseudo over-relaxation sweeps at gauge coupling $\beta=3.4$. At this coupling the lattice unit is $a\left(r_{0}\right)=0.102 \mathrm{fm}$ as determined from the Sommer parameter $r_{0}=0.49 \mathrm{fm}[9$. As it is well known, it is difficult to give an estimate on the systematic errors of $a\left(r_{0}\right)$ if the lattice is not fine. At this resolution, the systematic error should be on the percent level, while the statistical error is smaller than $1 \% 2$. The spatial extension of the lattice is $L \approx 1.8 \mathrm{fm}$. In our earlier results obtained at $L=1.8 \mathrm{fm}$ and $L=2.4 \mathrm{fm}$ at resolution $a=0.153 \mathrm{fm}$ we saw no finite size effects beyond the statistical errors. Our scaling analysis might be influenced by small finite size effects, however, and these physical effects might mix with the topological finite size artifacts as we discuss below.

Before summarizing our results, let us make two general remarks concerning scaling analyses in light hadron spectroscopy. Although the most interesting part of the spectrum for physics is where the quark masses go to their small physical values, the heavier quarks bring more information for scaling studies: the hadrons are more compact, they are more difficult to resolve and the cut-off effects are expected to be larger. In addition, the statistical and systematic errors are smaller, so the analysis is more conclusive.

The second remark refers to a special artifact of quenched light hadron spec- 
troscopy: the topological finite size artifacts. The discrete zero modes of the Dirac operator at $m_{q}=0$ are not suppressed in the quenched approximation and, in a finite volume at sufficiently small quark masses, they will corrupt the hadron propagators [10]. In ref. 1] we eliminated/reduced the topological finite size artifacts by using special hadron correlators in the pseudoscalar (PS), nucleon $(\mathrm{N})$ and delta $(\Delta)$ channels. Unfortunately, in the vector channel we did not find a natural solution for this problem and the artifacts turned out to be larger at $a\left(r_{0}\right)=0.102 \mathrm{fm}$ than at $a\left(r_{0}\right)=0.153 \mathrm{fm}$ in the same physical volume $L=1.8 \mathrm{fm}$. The effect is seen in the vector meson mass at the lightest quark masses in Fig. 1 where the dimensionless hadron masses are shown as the function of the dimensionless quark mass. This feature (namely, the bending down of the vector meson curve at small quark masses) is very similar to the artifacts we saw earlier appearing in smaller volumes, and then disappearing in our largest box with $L=2.4 \mathrm{fm}[1$. These quenching artifacts should be separated from the cut-off effects in a scaling test. This is relevant in particular in the vector channel, since the vector meson mass is used traditionally (unfortunately) as a unit for other hadron masses. The problem occurs at sufficiently light quark masses only, which gives an additional argument to avoiding this region in scaling studies.

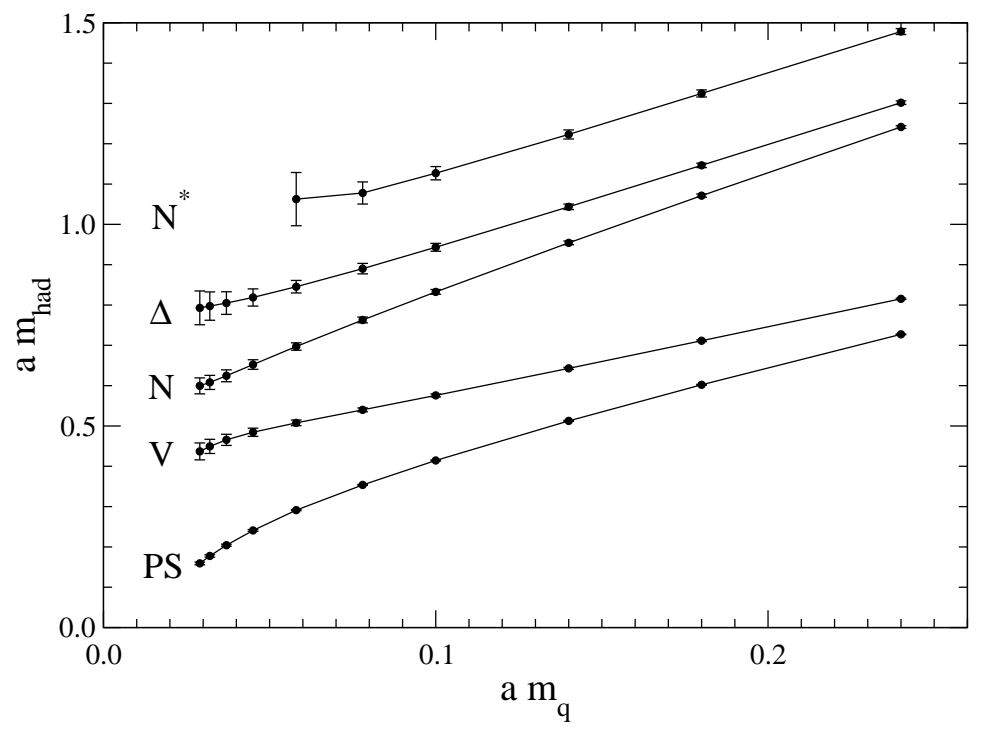

Figure 1: The hadron masses as the function of the quark mass in lattice units at $a=0.102 \mathrm{fm}$ in a box of size $L=1.8 \mathrm{fm}$.

We give here a brief summary of our conclusions. Considering mass ratios (like in the APE plot, or measuring the masses in units of a well determined fixed hadron mass) we see small cut-off effects in the baryon channel when comparing the results at $a=0.153$ and $0.102 \mathrm{fm}$ resolutions. The effect is seen for relative heavy quarks beyond the statistical errors. In the $N^{*}$ channel this is a several standard deviation effect and has a size of up to $\approx 2 \%$ in the ratio $m_{N^{*}} / m_{V}$, where $m_{V}$ is the vector meson mass. In the nucleon and delta channels the cut-off effects are smaller, but for heavy quarks they are also visible beyond the statistical errors. This small shift brings the FP results on the APE plot 
closer to and essentially consistent with the CP-PACS continuum extrapolated results with Wilson fermions [7. The improved staggered (asqtad[1]) data at $a=0.13 \mathrm{fm} 12$ with heavier quarks lie a few standard deviations higher and are close to the CP-PACS results at $a=0.05 \mathrm{fm}$. In addition, unlike at $a=0.153 \mathrm{fm}$, we see a discrepancy in the overall scale obtained in the gauge sector from the Sommer parameter $r_{0}$ and the scale from the spectrum of light hadrons. The hadron sector prefers a scale of $a=0.106(2) \mathrm{fm}$ as opposed to the scale of $a\left(r_{0}\right)=0.102 \mathrm{fm}$. In this case, however, it is difficult to estimate the systematic errors.

\section{Scaling analysis}

Concerning the details of the analysis we refer to ref. 11. In order to avoid very busy figures we shall use a few selected results only from other works in this paper. One can get a more complete picture by combining the figures here with those in ref. [1].

The measured new data are collected in Tables 1 and 2. Fig. 2 gives a quick overview on the cut-off effects in the different channels. In this figure the hadron masses are measured in $m_{\mathrm{V}}(0.75)$ units, where $m_{\mathrm{V}}(0.75)$ is the vector meson mass at $x=m_{\mathrm{PS}} / m_{\mathrm{V}}=0.75$. This figure indicates already that the change due to cut-off effects is small in $a \in(0.153-0.102) \mathrm{fm}$.

\begin{tabular}{c|c|c|c|c|c}
\hline$a m_{q}$ & $a m_{\mathrm{PS}}$ & $a m_{\mathrm{V}}$ & $a m_{\text {Oct }}(\mathrm{N})$ & $a m_{\text {Dec }}(\mathrm{N})$ & $a m_{\text {Oct }}\left(\mathrm{N}^{*}\right)$ \\
\hline 0.029 & $0.1592(33)$ & $0.437(21)$ & $0.599(20)$ & $0.793(42)$ & \\
0.032 & $0.1776(29)$ & $0.449(18)$ & $0.608(17)$ & $0.797(35)$ & \\
0.037 & $0.2042(25)$ & $0.466(14)$ & $0.624(15)$ & $0.805(28)$ & \\
0.045 & $0.2408(21)$ & $0.484(10)$ & $0.652(12)$ & $0.819(21)$ & \\
0.058 & $0.2913(14)$ & $0.508(7)$ & $0.697(9)$ & $0.845(15)$ & $1.063(66)$ \\
0.078 & $0.3537(13)$ & $0.540(5)$ & $0.763(7)$ & $0.890(13)$ & $1.078(27)$ \\
0.100 & $0.4144(12)$ & $0.576(4)$ & $0.833(6)$ & $0.943(10)$ & $1.127(17)$ \\
0.140 & $0.5127(11)$ & $0.643(3)$ & $0.954(5)$ & $1.044(7)$ & $1.223(11)$ \\
0.180 & $0.6021(10)$ & $0.711(2)$ & $1.071(4)$ & $1.146(5)$ & $1.325(9)$ \\
0.240 & $0.7272(9)$ & $0.815(2)$ & $1.241(3)$ & $1.302(4)$ & $1.478(7)$ \\
\hline
\end{tabular}

Table 1: The hadron masses for different quark masses in lattice units.

Fig. 3 shows $m_{N} / m_{V}$ as the function of $\left(m_{\mathrm{PS}} / m_{\mathrm{V}}\right)^{2}$ (APE plot). For the new $a\left(r_{0}\right)=0.102 \mathrm{fm} \mathrm{FP}$ data the 4 lightest points are not shown due to the problems in the vector meson channel discussed above. The remaining points lie slightly below of, but within the errors are consistent with our earlier $a\left(r_{0}\right)=$ $0.153 \mathrm{fm}$ results. If anything, the new data moved slightly towards the CPPACS continuum extrapolated Wilson data [7. This is illustrated also by Fig. [4 where $m_{\mathrm{N}}(x) / m_{\mathrm{V}}(0.75)$ is shown as the function of $a m_{\mathrm{V}}(0.75)$ for different fixed 


\begin{tabular}{c|c|c|c|c|c}
\hline$x$ & $\frac{m_{\mathrm{N}}(x)}{m_{\mathrm{V}}(x)}$ & $\frac{m_{\Delta}(x)}{m_{\mathrm{V}}(x)}$ & $\frac{m_{\mathrm{V}}(x)}{m_{\mathrm{V}}\left(x_{0}\right)}$ & $\frac{m_{\mathrm{N}}(x)}{m_{\mathrm{V}}\left(x_{0}\right)}$ & $\frac{m_{\Delta}(x)}{m_{\mathrm{V}}\left(x_{0}\right)}$ \\
\hline 0.40 & $1.351(64)$ & $1.769(97)$ & $0.753(37)$ & $1.018(27)$ & $1.333(54)$ \\
0.45 & $1.340(41)$ & $1.719(65)$ & $0.784(26)$ & $1.051(22)$ & $1.348(41)$ \\
0.50 & $1.347(27)$ & $1.689(44)$ & $0.810(18)$ & $1.092(18)$ & $1.368(32)$ \\
0.55 & $1.364(19)$ & $1.672(31)$ & $0.834(12)$ & $1.138(15)$ & $1.395(25)$ \\
0.60 & $1.385(14)$ & $1.659(23)$ & $0.864(8)$ & $1.196(12)$ & $1.433(20)$ \\
0.65 & $1.410(10)$ & $1.649(19)$ & $0.898(5)$ & $1.266(10)$ & $1.481(18)$ \\
0.70 & $1.436(8)$ & $1.641(14)$ & $0.940(2)$ & $1.350(7)$ & $1.543(13)$ \\
0.75 & $1.461(6)$ & $1.633(10)$ & $1.000(0)$ & $1.461(6)$ & $1.632(10)$ \\
0.80 & $1.486(5)$ & $1.623(7)$ & $1.079(2)$ & $1.602(6)$ & $1.750(10)$ \\
0.85 & $1.507(4)$ & $1.610(5)$ & $1.199(5)$ & $1.808(8)$ & $1.931(10)$ \\
\hline
\end{tabular}

Table 2: Hadron masses in $m_{\mathrm{V}}(x)$, or $m_{V}\left(x_{0}\right)$ units, where $x=m_{\mathrm{PS}} / m_{\mathrm{V}}$ and $x_{0}=0.75$.

$x=m_{\mathrm{PS}} / m_{\mathrm{V}}$ values. Here, $m_{\mathrm{V}}(0.75)$ is the vector meson mass at $x=0.75$ which is used as a convenient mass unit to measure the nucleon mass $m_{\mathrm{N}}(x)$. In this figure the CP-PACS data obtained in the $a=0.10-0.05 \mathrm{fm}$ range and their continuum extrapolations are compared to the FP data at $a\left(r_{0}\right)=0.153 \mathrm{fm}$ and $a\left(r_{0}\right)=0.102 \mathrm{fm}$. Also included in this plot are the ci results at $a\left(r_{0}\right)=0.148 \mathrm{fm}$. The Wilson action has large cut-off effects and the continuum extrapolation is a non-trivial task, even when the finest lattice spacing is $0.05 \mathrm{fm}$. Nonetheless, our results seem to support the continuum extrapolated CP-PACS numbers, in particular at $x=0.75$, where the errors are small. The $x=0.4$ new FP data on this figure should be taken cautiously due to the fact that $m_{N}(x=0.4)$ is indirectly influenced by the topological artifact problem in the vector channel. In fig. 3 the improved staggered (asqtad) data lie several standard deviations higher and are close to the CP-PACS Wilson action results at $a=0.05 \mathrm{fm}$.

Fig. [5 is an APE plot again where the FP data at $a\left(r_{0}\right)=0.153$ and $0.102 \mathrm{fm}$ are compared for the nucleon $(N)$, delta $(\Delta)$ and the negative parity baryon $N^{*}$. The nucleon, as we discussed above, and the $\Delta$ show some shift downwards as the lattice resolution is increased, but this effect is hardly statistically significant. This shift is, however, clearly seen for the $N^{*}$. We conclude that the FP action has an observable resolution error at $a\left(r_{0}\right)=0.153 \mathrm{fm}$ for this heavy, negative parity state.

Fig. [6 shows the hadron spectrum fixing the lattice unit and quark masses $a, m_{u d}$ and $m_{s}$ by $r_{0}, \pi, K$, or $\Phi$ (on the left) and by the $\pi, K$ and $\Phi$ mesons (on the right), respectively. Extrapolating (interpolating) the measured hadron masses to their physical points we followed the procedure in ref. [1] using functional forms dictated by quenched chiral perturbation theory [13]. On the r.h.s. the stable $N$ and narrow $\Omega$ are at the right place, whereas the broad $\Delta$ and $N^{*}$ are pushed upwards. The $\rho$ is also at the correct place, but only by accident since the vector meson mass is pushed down by the topological finite size artifacts at light quark masses as we discussed before. The fitted value of the 


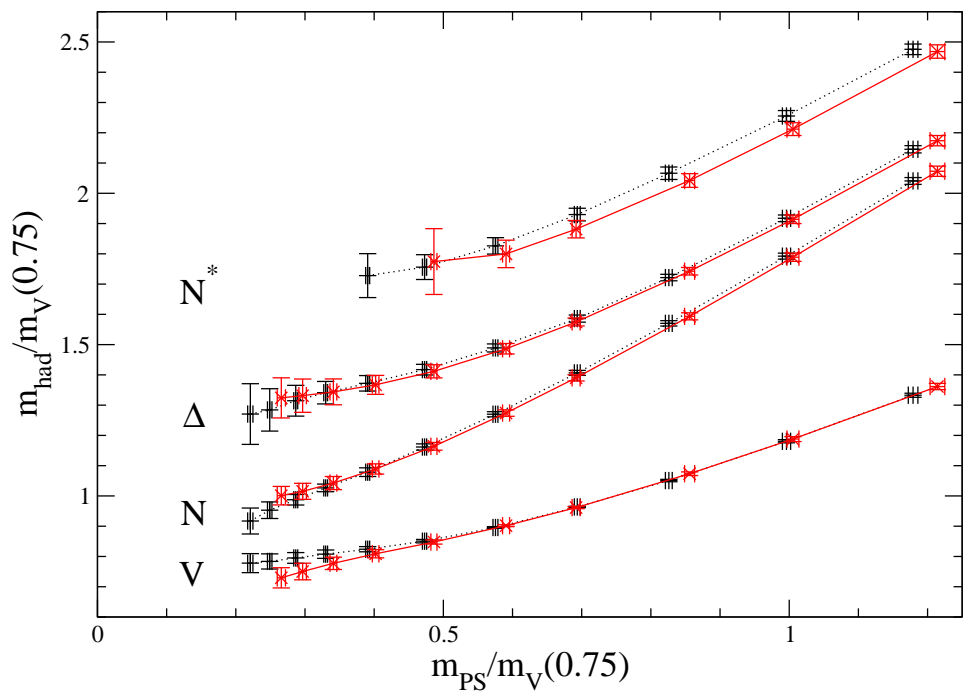

Figure 2: The hadron masses in the vector meson, nucleon, $\Delta$ and $N^{*}$ channels as the function of the pseudoscalar mass. All the masses are measured in $m_{\mathrm{V}}(0.75)$ units. Here $m_{\mathrm{V}}(0.75)$ is the vector meson mass at a quark mass value, where $x=m_{\mathrm{PS}} / m_{\mathrm{V}}=0.75$. The points connected by dotted and continuous lines refer to $a\left(r_{0}\right)=0.153 \mathrm{fm}$ and $0.102 \mathrm{fm}$, respectively.

lattice unit $a=0.1055$ (14) fm (the error is statistical only) deviates from the scale found in the gauge sector $a\left(r_{0}\right)=0.102 \mathrm{fm}$ (error on the percent level). On the l.h.s. the scale is taken from $r_{0}$ in the gauge sector. This spectrum is somewhat distorted which comes from the difference between the preferred scale in the hadron spectrum and in the gauge sector from $r_{0}$.

In Fig. 17 the product $r_{0} m_{V}(0.75)$ is shown as the function of $a / r_{0}$ with $r_{0}$ from the gauge sector. The new data confirm the trend indicated by the $L=$ $1.2 \mathrm{fm}$ data in ref. 11: $r_{0} m_{V}(0.75)$ is increasing as we go towards the continuum limit. This effect is seen in the new data beyond the statistical errors. The value at $a\left(r_{0}\right)=0.102 \mathrm{fm}$ seems to be consistent with the continuum extrapolated CPPACS number.

\section{Pion Scattering Length}

The pion scattering length (in the isospin 2 channel) was studied as an application other than standard hadron spectroscopy. We use the finite volume method of Lüscher [14] to relate the energy shift of two pions in a finite volume to the infinite volume scattering length,

$$
\delta E=-\frac{4 \pi a_{0}}{m_{\mathrm{PS}} L^{3}}\left\{1+c_{1} \frac{a_{0}}{L}+c_{2} \frac{a_{0}^{2}}{L^{2}}\right\}+\ldots
$$




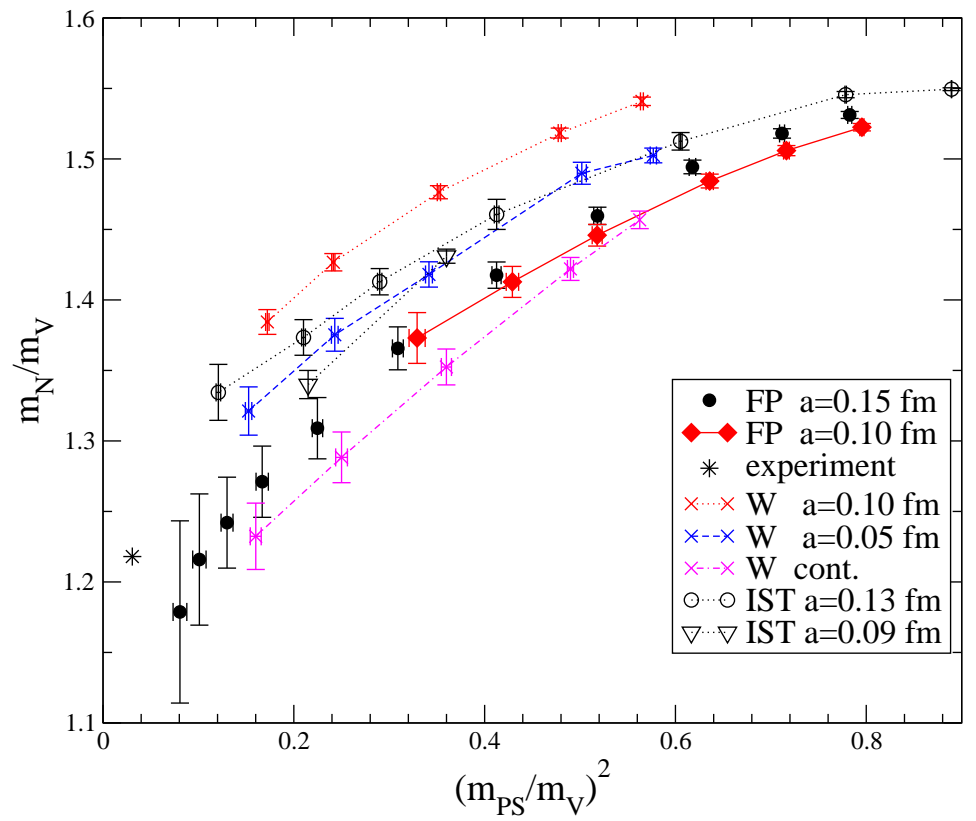

Figure 3: APE plot, where the FP numbers are compared to the unimproved Wilson fermion data and their continuum extrapolation. The improved staggered (asqtad) results are shown also.

where $c_{1}=-2.837297$ and $c_{2}=6.375183$. The fitting procedure is described in ref. [6].

Cutoff effects were studied in the small volume $(1.2 \mathrm{fm})^{3}$ using all three different lattice spacings $(0.08,0.10,0.15 \mathrm{fm})$ and in an intermediate volume of $(1.8 \mathrm{fm})^{3}$ with $\mathrm{a} \sim 0.10$ and $0.15 \mathrm{fm}$. A wide range of quark masses were studied, with the smallest quark mass resulting in $m_{\mathrm{PS}} / m_{\mathrm{V}} \sim 0.35$. In this quenched study, we keep those masses with $m_{\mathrm{PS}} L \gtrsim 4$.

In the small volume, the translation of the energy shift to the scattering length may involve large corrections and so it is preferable to simply compare the energy shifts rather than the scattering lengths. In Fig. [8 we show the energy shift of the two pion state in the $(1.2 \mathrm{fm})^{3}$ box for all three different lattice spacings. The energy shift below a mass of $1 \mathrm{GeV}$ shows scaling within the errors from the three different lattice spacings.

For the intermediate volume, $(1.8 \mathrm{fm})^{3}$, we calculate the scattering length from Lüscher's formula up to $\mathcal{O}\left(1 / L^{5}\right)$ where the pseudoscalar mass satisfies $m_{\mathrm{PS}} L \gtrsim 4$. The dimensionless quantity $a_{0} m_{\mathrm{PS}}$ is shown in Fig. 9 Scaling is observed in this quantity below a pseudoscalar mass of $\sim 1.2 \mathrm{GeV}$. For masses greater than $1.2 \mathrm{GeV}$, it is possible that some scaling violations appear, but the differences are still consistent within the statistical errors. We include in Fig. 9 the results from the largest volume to show that the truncation at $1 / L^{5}$ is a negligible effect and that the extracted scattering length is indeed a physical quantity (within the quenched approximation). The scattering lengths are listed 


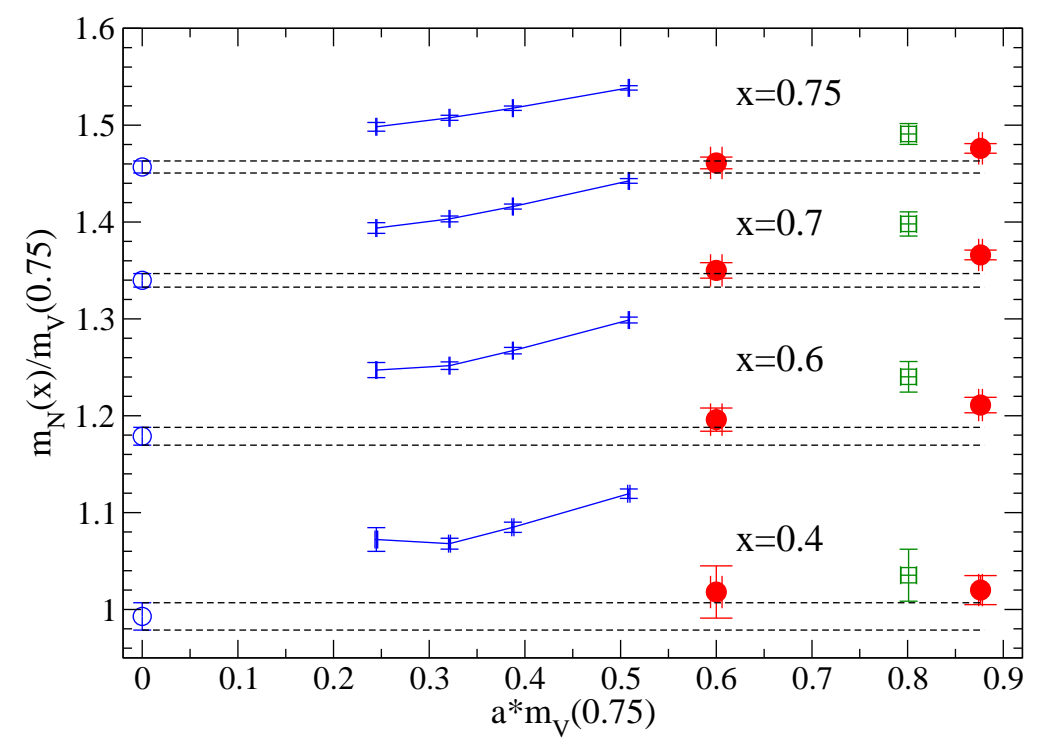

Figure 4: The cut-off dependence of the nucleon mass in $m_{\mathrm{V}}(0.75)$ units for different $x=m_{\mathrm{PS}} / m_{\mathrm{V}}$ values. The CP-PACS Wilson data (4 points for $a \in$ $(0.10,0.05) \mathrm{fm}$ and their continuum extrapolation (circles at $a=0$ ), the FP numbers at $a=0.153$ and $a=0.102 \mathrm{fm}$ and the CI results at $a=0.148 \mathrm{fm}$ (squares) are compared in this figure.

\section{in Tables 35}

We compare our results from large and intermediate volumes to other large scale simulations using the Wilson action. The comparison requires some care as the volumes used are not always the same. However, we have seen that the truncation is under control at least if the volume is greater than $(1.8 \mathrm{fm})^{3}$. The physical quantity that remains finite in the chiral limit, $a_{0} / m_{\mathrm{PS}}$, is plotted against $m_{\mathrm{PS}}$ in physical units in Fig. 10. We note that, in addition to the agreement of the $a=0.15 \mathrm{fm}$ and $a=0.10 \mathrm{fm}$ results, all of our scattering lengths are statistically consistent with the finest lattice results from the JLQCD collaboration 17 who use the standard Wilson action at $\beta=6.3$. We conclude that the cutoff effects of the FP action on the pion scattering length are small at nearly twice the lattice spacings required for unimproved actions. This gain is important for such quantities where a chiral action in a large volume is needed. 


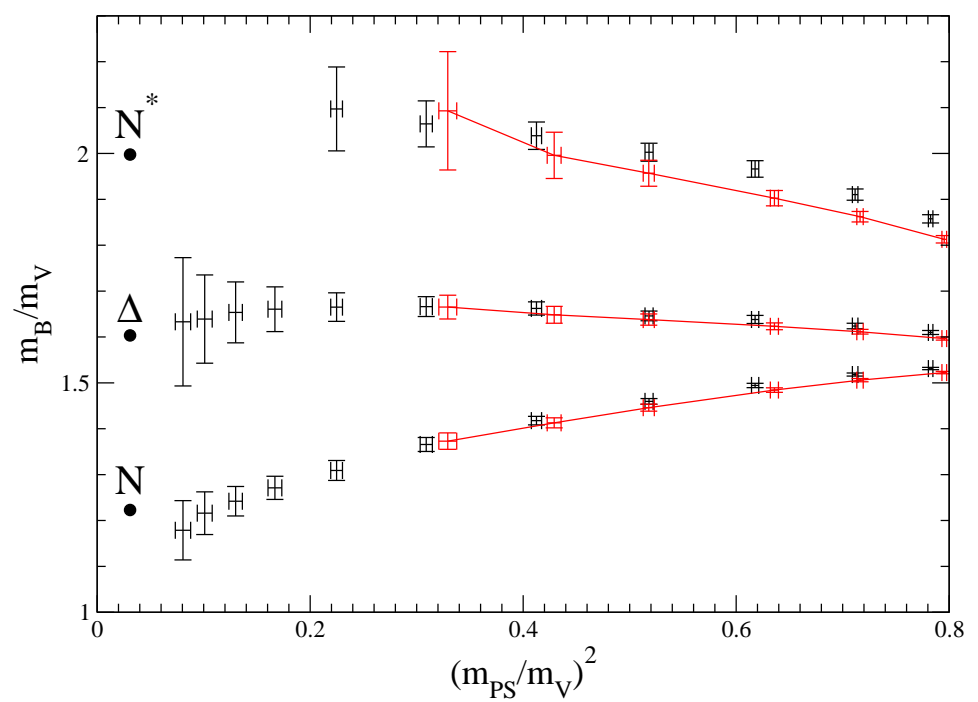

Figure 5: APE plot with the FP results at $a=0.102 \mathrm{fm}$ (connected by a continuous line) and $a=0.153 \mathrm{fm}$ in the $N, \Delta$ and $N^{*}$ channels.

\section{SUMMARY}

In this work we discussed the cut-off effects in simulations with the parametrized FP action. For this purpose going deep in the chiral limit is not very important. It is good to see, nevertheless, that with this action one can reproduce cleanly the divergence $\propto \ln (m a)$ in $\left(a m_{\mathrm{PS}}\right)^{2} /(a m)$ predicted by quenched chiral perturbation theory [13] as shown in Fig. 11] Here $m=m_{q}+m_{\text {res }}$, where $m_{q}$ is the quark mass in the simulation and $m_{\text {res }}$ is the residual additive mass renormalization due to the parametrization error of the FP Dirac operator.

\begin{tabular}{c|c|c|c|c}
\hline$a m_{q}$ & $a \delta E$ & $a_{0} / a$ & $a_{0} /\left(a^{2} m_{\mathrm{PS}}\right)$ & $a_{0} m_{\mathrm{PS}}$ \\
\hline 0.33 & $0.0041(10)$ & $-1.12(22)$ & $-1.10(22)$ & $-1.15(23)$ \\
0.25 & $0.0049(10)$ & $-1.12(19)$ & $-1.30(22)$ & $-0.97(16)$ \\
0.18 & $0.00597(97)$ & $-1.13(15)$ & $-1.58(21)$ & $-0.81(11)$ \\
0.13 & $0.00691(89)$ & $-1.11(12)$ & $-1.84(20)$ & $-0.666(72)$ \\
0.09 & $0.00733(90)$ & $-0.99(10)$ & $-1.99(20)$ & $-0.497(50)$ \\
0.06 & $0.0085(10)$ & $-0.958(97)$ & $-2.33(23)$ & $-0.395(40)$ \\
0.04 & $0.0081(15)$ & $-0.78(13)$ & $-2.30(38)$ & $-0.264(43)$ \\
0.028 & $0.0089(19)$ & $-0.73(14)$ & $-2.54(49)$ & $-0.209(40)$ \\
0.021 & $0.0103(21)$ & $-0.73(13)$ & $-2.93(54)$ & $-0.182(33)$ \\
\hline
\end{tabular}

Table 3: The scattering lengths for the $\beta=3.0,16^{3} \times 32$ lattice. 


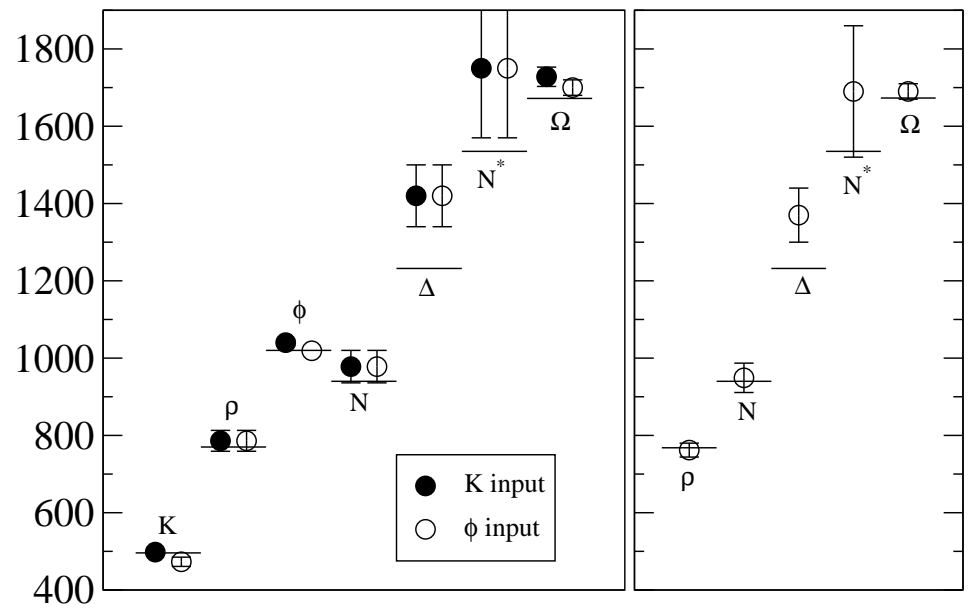

Figure 6: Hadron masses in $\mathrm{MeV}$ units. The lattice unit and the quark masses $a, m_{u d}$ and $m_{s}$ are fixed by $r_{0}, \pi, K$, or $\Phi$ (on the left) and by the $\pi, K$ and $\Phi$ mesons (on the right), respectively.

\begin{tabular}{c|c|c|c|c}
\hline$a m_{q}$ & $a \delta E$ & $a_{0} / a$ & $a_{0} /\left(a^{2} m_{\mathrm{PS}}\right)$ & $a_{0} m_{\mathrm{PS}}$ \\
\hline 0.33 & $0.01021(49)$ & $-1.096(40)$ & $-1.070(40)$ & $-1.123(41)$ \\
0.25 & $0.01232(52)$ & $-1.113(37)$ & $-1.285(43)$ & $-0.964(31)$ \\
0.18 & $0.01466(59)$ & $-1.103(35)$ & $-1.533(48)$ & $-0.793(25)$ \\
0.13 & $0.01660(76)$ & $-1.062(38)$ & $-1.755(64)$ & $-0.642(23)$ \\
0.09 & $0.0164(13)$ & $-0.908(57)$ & $-1.81(11)$ & $-0.457(29)$ \\
\hline
\end{tabular}

Table 4: The scattering lengths for the $\beta=3.0,12^{3} \times 24$ lattice.

Acknowledgements We thank Christine Davies for the correspondence on the interpretation of the APE plot. We thank also the discussions with Anna Hasenfratz and the members of the BGR Collaboration, and the support from the Swiss Center for Scientific Computing in Manno, where the numerical simulations were done. K.J.J. would like to thank Colin Morningstar for some of the fitting routines used in the analysis of the scattering length. This work was supported by the Schweizerischer Nationalfonds. 


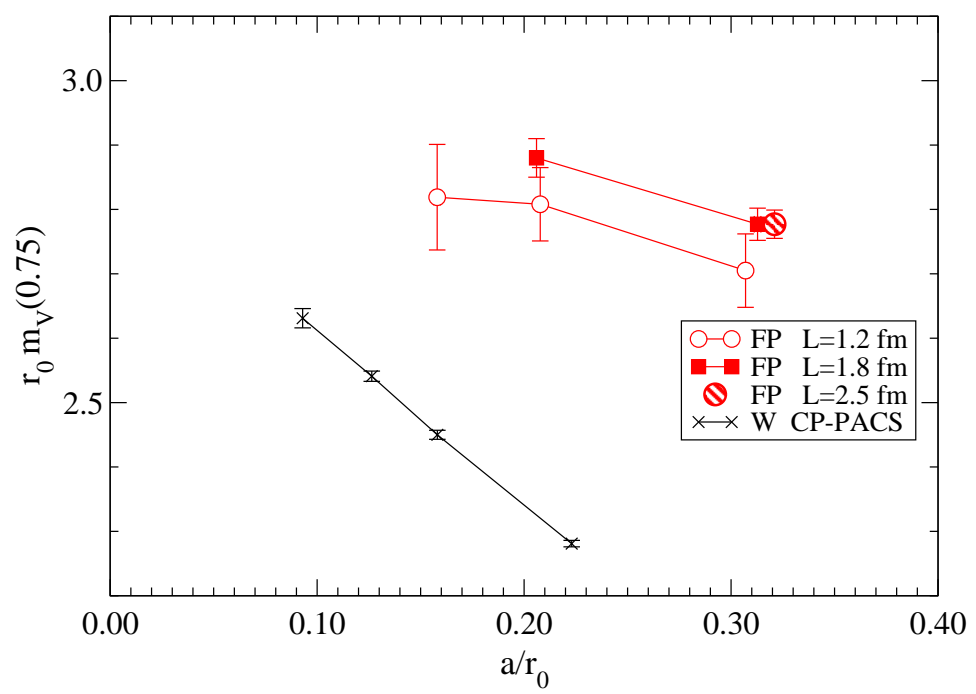

Figure 7: Scaling behavior of $m_{V}(0.75)$ in $1 / r_{0}$ unit.

\begin{tabular}{c|c|c|c|c}
\hline$a m_{q}$ & $a \delta E$ & $a_{0} / a$ & $a_{0} /\left(a^{2} m_{\mathrm{PS}}\right)$ & $a_{0} m_{\mathrm{PS}}$ \\
\hline 0.240 & $0.00562(56)$ & $-1.49(12)$ & $-2.04(16)$ & $-1.080(86)$ \\
0.180 & $0.00728(58)$ & $-1.57(10)$ & $-2.61(16)$ & $-0.945(60)$ \\
0.140 & $0.00877(69)$ & $-1.60(10)$ & $-3.12(19)$ & $-0.821(51)$ \\
0.100 & $0.01062(85)$ & $-1.576(99)$ & $-3.80(24)$ & $-0.653(42)$ \\
0.078 & $0.0117(10)$ & $-1.50(10)$ & $-4.25(28)$ & $-0.533(35)$ \\
0.058 & $0.0126(14)$ & $-1.36(12)$ & $-4.66(43)$ & $-0.398(36)$ \\
0.045 & $0.0160(55)$ & $-1.43(40)$ & $-5.9(1.7)$ & $-0.348(97)$ \\
0.037 & $0.0191(96)$ & $-1.45(60)$ & $-7.0(2.8)$ & $-0.30(12)$ \\
\hline
\end{tabular}

Table 5: The scattering lengths for the $\beta=3.4,18^{3} \times 36$ lattice. 


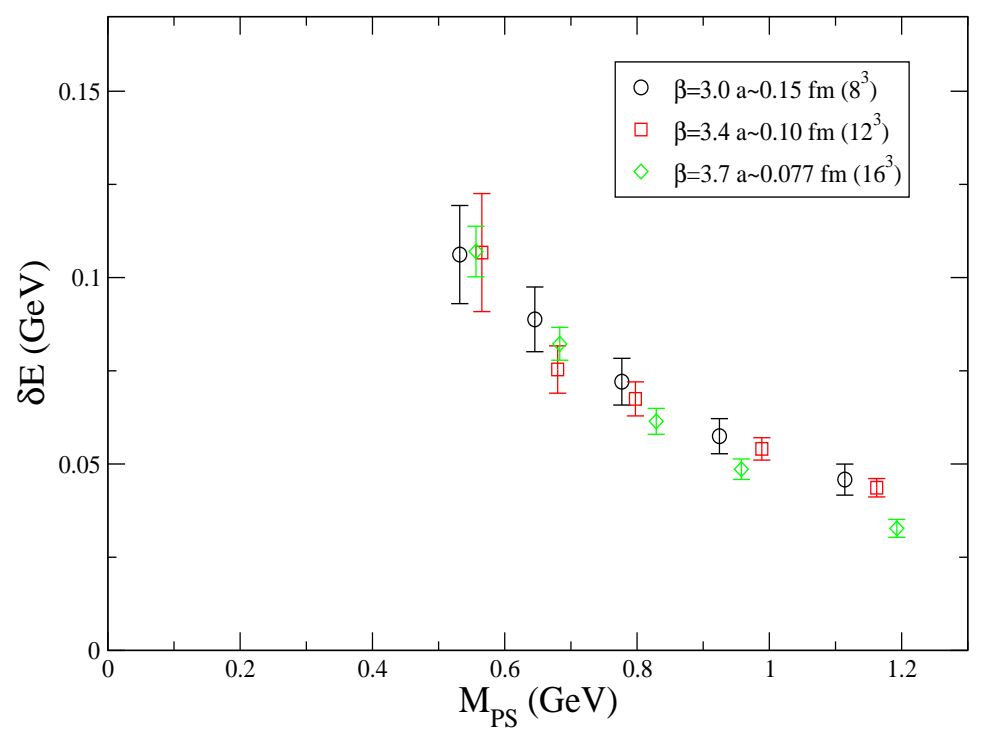

Figure 8: The energy shift of the two pion state in the $(1.2 \mathrm{fm})^{3}$ box for the three different lattice spacings.

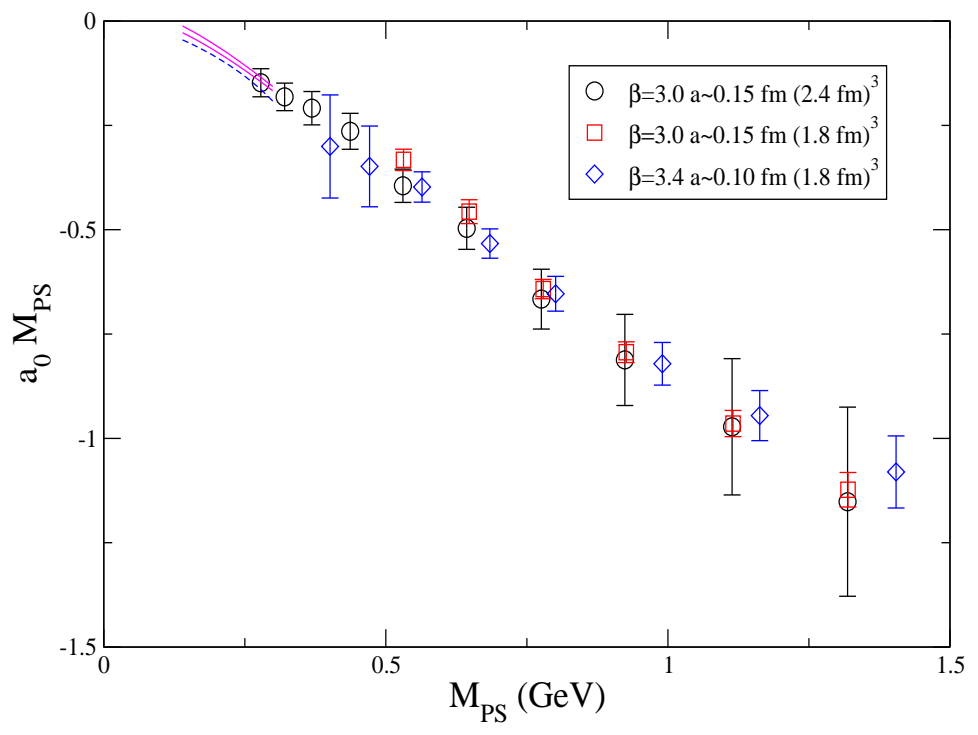

Figure 9: The dimensionless scattering length in the $(1.8 \mathrm{fm})^{3}$ box for two different lattice spacings, $\mathrm{a}=0.10 \mathrm{fm}$ and $\mathrm{a}=0.15 \mathrm{fm}$. Chiral perturbation theory curves (15, 19]) are shown for $m_{\mathrm{PS}}<0.3 \mathrm{GeV}$. 


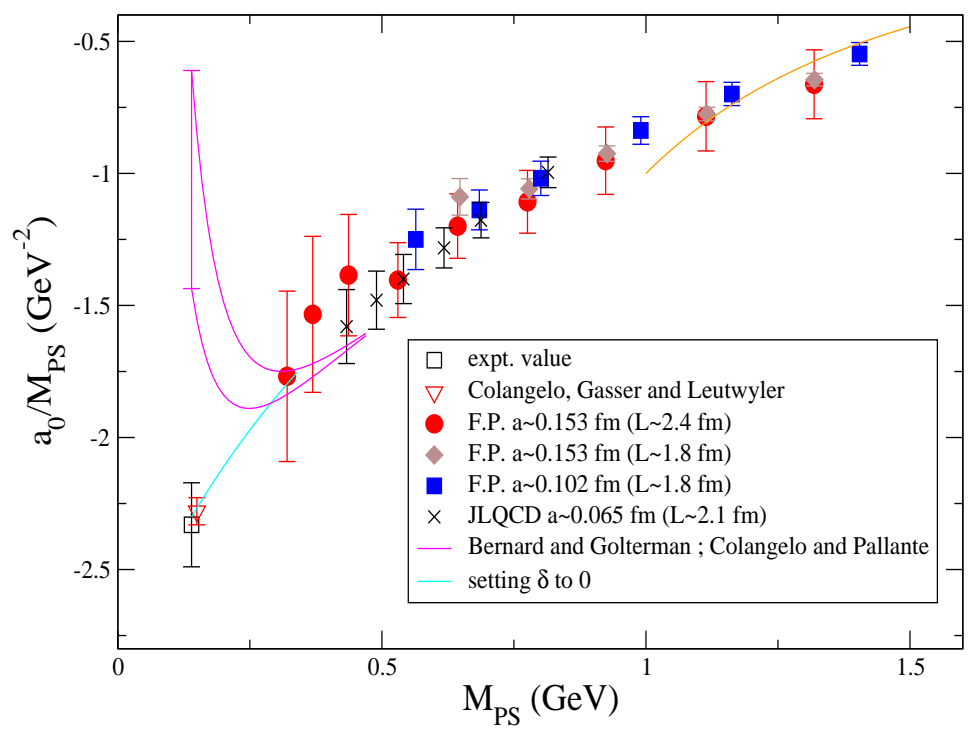

Figure 10: Summary of all the intermediate and large volume results together with the the Wilson action results (17]) for the ratio $a_{0} / m_{\mathrm{PS}}$. NNLO chiral perturbation theory results ([19]) and NLO quenched chiral p.t. curves (15]) are shown with the measured values for $F_{\pi}$. The behaviour expected from the scattering of hard spheres are shown at large $m_{\mathrm{PS}}$.

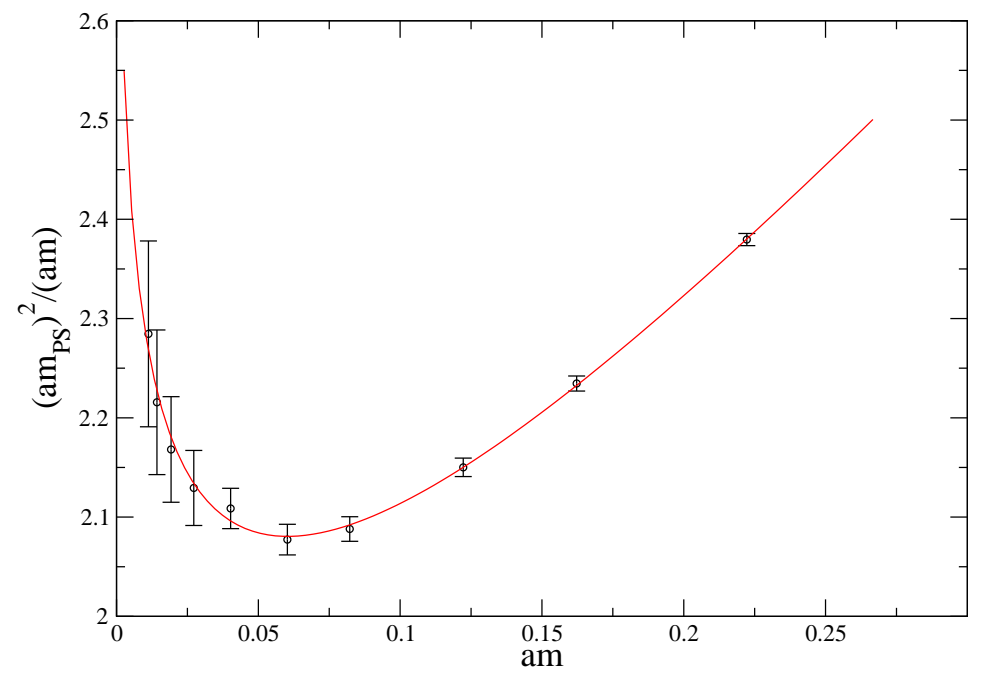

Figure 11: The ratio $\left(a m_{\mathrm{PS}}\right)^{2} /(a m)$ at fixed $a=0.102 \mathrm{fm}$ as the function of the quark mass $a m, m=m_{q}+m_{\text {res }}$, where $m_{\text {res }}$ is the residual additive renormalization due to the parametrization error of the FP Dirac operator. The continuous line is a fit of the form $\alpha+\beta \ln (a m)+\gamma a m$ suggested by quenched chiral perturbation theory. 


\section{References}

[1] C. Gattringer et al., BGR-Collaboration, Nucl. Phys. B 677 (2004) 3, hep-lat/0307013.

[2] F. Niedermayer, P. Rüfenacht and U. Wenger, Nucl. Phys. B 597 (2001) 413, hep-lat/0007007

[3] P. Hasenfratz, S. Hauswirth, K. Holland T. Jörg, F. Niedermayer and U. Wenger, Int. J. Mod. Phys. C 12 (2001) 691, hep-lat/0003013

[4] M. Lüscher and P. Weisz, Commun. Math. Phys. 97 (1985) 59; Commun. Math. Phys. 98 (1985) 433, Erratum.

[5] C. Gattringer, Phys. Rev. D 63 (2001) 114501, hep-lat/0003005 C. Gatringer, I. Hip and C. B. Lang, Nucl. Phys. B 597 (2001) 451, hep-lat/0007042

[6] K. J. Juge, Nucl. Phys. Proc. Suppl. 129 (2004) 194, hep-lat/0309075

[7] CP-PACS Collaboration, S. Aoki, et al., Phys. Rev. D 67 (2003) 034503, hep-lat/0206009.

[8] C. T. H. Davies, G. P. Lepage, F. Niedermayer and D. Toussaint, hep-lat/0409039

[9] R. Sommer, Nucl. Phys. B 411 (1994) 839, hep-lat/9310022

[10] T. Blum, et al., Phys. Rev. D 69 (2004) 074502, hep-lat/0007038, L. Giusti, C. Hoelbling and C. Rebbi, Phys. Rev. D 64 (2001) 114508, hep-lat/0108007.

[11] K. Orginos and D. Toussaint, Phys. Rev. D 59 (1999) 014501, hep-lat/9805009 G. P. Lepage, Phys. Rev. D 59 (1999) 074502, hep-lat/9809157 K. Orginos, R. L. Sugar and D. Toussaint,Phys. Rev. D 60 (1999) 054503, hep-lat/9903032 C. T. H. Davies et al., Phys. Rev. Lett. 92 (2004) 022001, hep-lat/0304004 and references therein.

[12] D. Toussaint, Nucl. Phys. B Proc. Suppl. 106 (2002) 111, hep-lat/0110010 C. Bernard et al., hep-lat/0208041.

[13] C. Bernard and M. Golterman, Phys. Rev. D 46 (1992) 853, hep-lat/9204007 S. R. Sharpe, Phys. Rev. D 46 (1992) 3146, hep-lat/9205020 CP-PACS Collaboration, T. Yoshié, Prog. Theor. Phys. 105 (2001) 37

[14] M. Lüscher, Commun. Math. Phys. 104 (1986) 177; 105 (1986) 153; Nucl. Phys. B 354 (1991) 531.

[15] C. Bernard and M. Golterman, Phys. Rev. D 53 (1996) 476, hep-lat/9507004 G. Colangelo and E. Pallante, Nucl. Phys. B 520 (1998) 433, hep-lat/9708005

[16] C. Liu, J. Zhang, Y. Chen and J. Ma, Nucl. Phys. B 624 (2002) 360, hep-lat/0109020. 
[17] JLQCD Collaboration (S. Aoki et al.), Phys. Rev. D 66 (2002) 077501, hep-lat/0206011.

[18] CP-PACS Collaboration (S. Aoki et al.), Phys. Rev. D 67 (2003) 014502, hep-lat/0209124.

[19] G. Colangelo, J. Gasser and H. Leutwyler, Nucl. Phys. B 603 (2001) 125, hep-ph/0103088 